\title{
Impactos de sistemas eletrônicos de comunicação entre a atenção primária e a especializada: uma revisão integrativa
}

\author{
Impacts of electronic communication systems between primary and specialized care: an integrative \\ review
}

Impactos de los sistemas de comunicaciones electrónicas entre atención primaria y especializada: una revisión integrativa

Recebido: 26/07/2021 | Revisado: 31/07/2021 | Aceito: 02/08/2021 | Publicado: 08/08/2021

\author{
Marcella Cristina Halliday Muniz \\ ORCID: https://orcid.org/0000-0003-2126-5457 \\ Universidade Federal de Sergipe, Brasil \\ E-mail: marcellahalliday@ hotmail.com \\ Mario Adriano dos Santos \\ ORCID: https://orcid.org/0000-0002-5721-6115 \\ Universidade Federal de Sergipe, Brasil \\ E-mail: mario@alergia.org \\ Brenda Carla Lima Araújo \\ ORCID: https://orcid.org/0000-0002-4720-1710 \\ Universidade Federal de Sergipe, Brasil \\ E-mail: brendaaraujo@yahoo.com.br \\ Adicinéia Aparecida de Oliveira \\ ORCID: https://orcid.org/0000-0002-1551-1992 \\ Universidade Federal de Sergipe, Brasil \\ E-mail: adicineia @gmail.com \\ Silvia de Magalhães Simões \\ ORCID: https://orcid.org/0000-0003-2751-7993 \\ Universidade Federal de Sergipe, Brasil \\ E-mail: silviasimoes@gmail.com
}

\section{Resumo}

Plataformas de comunicação interprofissional têm se mostrado promissoras na articulação entre a atenção primária e a especializada. Este artigo buscou analisar os impactos na saúde resultantes do uso de sistemas eletrônicos de comunicação entre médicos generalistas e especialistas. Foi realizada uma revisão integrativa nas bases de dados BVS, PubMed e Scielo, e uma análise da literatura cinza na plataforma Google Scholar, sem filtro de tempo. Após aplicação dos critérios de inclusão e exclusão, foram selecionados 27 estudos. Estes foram publicados entre os anos de 2000 e 2020, foram em sua maioria estudos canadenses, e tiveram os sistemas eletrônicos desenvolvidos para múltiplas especialidades como predominantes. Por meio de abordagens variadas, os artigos destacaram redução nas taxas de encaminhamento para serviços de atenção especializada, economia de custos e melhoria da qualidade da assistência em saúde prestada pela atenção primária como impactos resultantes do uso das plataformas de comunicação interprofissional.

Palavras-chave: Consulta remota; Acesso aos serviços de saúde; Atenção primária à saúde; Atenção secundária à saúde; Encaminhamento e consulta.

\begin{abstract}
Interprofessional communication platforms have shown promise in the articulation between primary and specialized care. This article sought to analyze the health impacts resulting from the use of electronic communication systems between general practitioners and specialists. An integrative review was carried out in the BVS, PubMed and Scielo databases, and an analysis of gray literature in the Google Scholar platform, without a time filter. After applying the inclusion and exclusion criteria, 27 studies were selected. These were published between 2000 and 2020, were mostly Canadian studies, and had electronic systems developed for multiple specialties as predominant. Through a variety of approaches, the articles highlighted a reduction in referral rates to specialized care services, cost savings and improvement in the quality of health care provided by primary care as impacts resulting from the use of interprofessional communication platforms.
\end{abstract}

Keywords: Remote consultation; Health services accessibility; Primary health care; Secondary care; Referral and consultation. 


\section{Resumen}

Las plataformas de comunicación interprofesional se han mostrado prometedoras en la articulación entre atención primaria y especializada. Este artículo buscó analizar los impactos en la salud derivados del uso de sistemas de comunicación electrónica entre médicos generalistas y especialistas. Se realizó una revisión integradora en las bases de datos BVS, PubMed y Scielo, y un análisis de la literatura gris en la plataforma Google Scholar, sin filtro de tiempo. Después de aplicar los criterios de inclusión y exclusión, se seleccionaron 27 estudios. Estos se publicaron entre 2000 y 2020, eran en su mayoría estudios canadienses y predominaban los sistemas electrónicos desarrollados para múltiples especialidades. A través de una variedad de enfoques, los artículos destacaron una reducción en las tasas de derivación a servicios de atención especializada, el ahorro de costos y la mejora en la calidad de la atención médica brindada por la atención primaria como impactos resultantes del uso de plataformas de comunicación interprofesional.

Palabras clave: Consulta remota; Accesibilidad a los servicios de salud; Atención primaria de salud; Atención secundaria de salud; Derivación y consulta.

\section{Introdução}

A Atenção Primária em Saúde (APS) constitui o primeiro nível e a porta de entrada da rede assistencial em saúde no Brasil. À APS cabe o papel de receber o usuário como ponto inicial de contato, organizando e coordenando os cuidados prestados dentro da rede de assistência, e referenciando para o nível subsequente, a atenção especializada (AE), os casos nos quais se pretenda obter uma avaliação diagnóstica específica ou a otimização de um esquema terapêutico (Bernardino Junior et al., 2020). Considerando a atenção à saúde como um processo contínuo de cuidados, subentende-se o quão fundamental é, para a garantia de acesso pleno e irrestrito ao sistema, a existência de instrumentos de integração entre seus elementos constituintes.

A entrega de cobertura e acesso universal aos serviços de saúde relaciona-se à continuidade da atenção' que, por sua vez, vincula-se diretamente ao atributo de coordenação de cuidados da APS, por meio do qual há o reconhecimento dos problemas de saúde que requerem seguimento e a efetiva ordenação dos fluxos e contrafluxos de pessoas, produtos e informações, capitaneados pela própria APS, ao longo de todos os pontos de atenção à saúde (Bernardino Junior et al., 2020; Organização Pan-Americana da Saúde [OPAS], 2011).

A despeito da ampla entrega de serviços de cuidados primários no território brasileiro ao longo dos últimos anos, a articulação entre este primeiro ponto de contato dos indivíduos e os demais níveis da rede assistencial permanece incipiente, especialmente no que tange ao acesso à AE, considerada o grande gargalo do nosso sistema público de saúde (Almeida, Gérvas, Freire, \& Giovanella, 2013), acumulando longas filas de espera e demanda reprimida. A ampliação da cobertura da atenção básica e a longevidade populacional mais ampla e atrelada a uma maior prevalência de doenças crônicas são apontadas como condições potencializadoras do aumento da demanda para a AE (Farias, Giovanella, Oliveira, \& Santos Neto, 2019).

Os impactos relacionados a um tempo de espera excessivo para avaliação em serviços especializados se traduzem em atrasos em diagnósticos, diminuição da efetividade dos tratamentos e aumento da ansiedade por parte dos pacientes (Farias et al., 2019; Liddy et al., 2019a). Neste contexto, a literatura científica traz relatos de prestação remota de cuidados em saúde utilizando tecnologia, através de plataformas baseadas na world wide web (web-based) ou aplicativos para telefones móveis, a fim de preencher a lacuna na acessibilidade dentro da rede assistencial. A estratégia conhecida pelo termo em inglês Electronic Consultation consiste em sistemas de consulta eletrônica nos quais dispositivos inovadores no campo da saúde digital conectam diretamente profissionais da atenção básica e especialistas, permitindo a troca de informações e aconselhamento de manejo de casos clínicos, reduzindo a necessidade de atendimento presencial junto à AE e melhorando a comunicação interprofissional (Liddy et al., 2016a; Ray \& Kahn, 2020; Tuot et al., 2018;). Ganhos de eficiência na prestação de cuidados especializados, redução de custos por meio da restrição de encaminhamentos desnecessários e melhoria na capacidade de tomada de decisão do profissional da atenção primária, são ainda citadas como vantagens desses sistemas (Knox, Murphy, Leslie, Wick, \& Tuot, 2020; Olayiwola et al., 2019;), para além dos benefícios na esfera educacional inerente a este tipo de interação entre pares.

Potencialmente transformadora desta realidade de demanda reprimida são os serviços oferecidos pelas plataformas de Telessaúde, que despontam como soluções capazes de otimizar o acesso, melhorar a qualidade e reduzir os custos assistenciais 
em condições tão diversas em estrutura e financiamento no setor saúde, em um país de dimensões continentais como o Brasil (Ministério da Saúde, n.d.).

Recente revisão integrativa acerca das contribuições do Programa Nacional de Telessaúde na formação de recursos humanos na atenção primária, destacou experiências exitosas de núcleos de telessaúde de diversos estados do Brasil, com ênfase à grande efetividade das ferramentas ofertadas pelo Programa na capacitação e qualificação profissional, refletindo em um aprimoramento das práticas assistenciais no ambiente da APS, melhor desempenho e maior resolutividade neste nível, quando ao assumir o protagonismo na tomada de decisão, contribui para ampliar a acessibilidade da população a uma atenção integral em saúde (Belber, Passos, Borysow, \& Maeyama, 2021).

O objetivo desta revisão integrativa foi identificar as evidências científicas disponíveis acerca dos impactos na saúde resultantes da utilização de sistemas eletrônicos de comunicação entre profissionais generalistas atuantes em cuidados primários e aqueles atuantes na $\mathrm{AE}$.

\section{Metodologia}

Pesquisa baseada na metodologia de revisão integrativa, cumprindo as seguintes etapas: 1- elaboração da pergunta norteadora e decisão da estratégia de busca; 2- estabelecimento dos critérios de inclusão e exclusão dos estudos; 3- identificação dos estudos selecionados; 4- categorização dos estudos incluídos com base nas principais informações que serão extraídas; 5 interpretação dos resultados; 6- sumário do conhecimento obtido (Botelho, Cunha, \& Macedo, 2011).

A pergunta norteadora da presente revisão integrativa foi: "Qual o impacto na saúde resultante do uso de sistemas eletrônicos de comunicação entre profissionais que atuam em cuidados primários e na atenção especializada?”

Para levantamento dos artigos foi realizada uma busca nas seguintes bases de dados: Biblioteca Virtual em Saúde (BVS), PubMed e Scientific Electronic Library Online (Scielo). A pesquisa na literatura cinza foi realizada nos cem primeiros artigos recuperados pela plataforma Google Scholar. O processo de rastreamento e análise dos artigos ocorreu durante os meses de fevereiro e julho de 2021. A expressão de busca utilizada foi composta por termos indexados nos vocabulários estruturados Medical Subject Headings (MeSH) e Descritores em Ciências da Saúde (DeCS), com a inclusão da terminologia "Electronic Consultation" pelo uso comum, tendo em vista a mesma não integrar os referidos vocabulários. A estratégia de busca utilizada foi:

\footnotetext{
("Primary Health Care" OR "Physicians, Primary Care" OR "General Practitioners" OR "Secondary Care" OR "Interprofessional Relations") AND ("Mobile Applications" OR "Mobile App" OR "Remote Consultation" OR Teleconsultation OR "Electronic Consultation") AND ("Referral and Consultation" OR "Second Opinion")
}

Os critérios de inclusão determinados para a seleção dos artigos foram: artigos disponíveis integralmente, que abordem projetos de sistemas eletrônicos (aplicativos para telefones móveis ou plataformas web-based) concebidos para uso entre profissionais de serviços de saúde, sem filtros de idioma e tempo. Foram incluídos estudos originais transversais e longitudinais. Foram excluídos da seleção, artigos abordando sistemas de videoconferência envolvendo médicos e a participação conjunta dos pacientes, artigos de revisão, diretrizes clínicas, editoriais e estudos duplicados.

Para a análise dos dados dos artigos selecionados foi utilizada uma planilha constando os seguintes itens: título do artigo, ano, país de origem, autor principal, objetivos, características metodológicas do estudo e principais resultados encontrados. 


\section{Resultados}

A busca inicial contabilizou 376 artigos distribuídos nas bases de dados, sendo 48 artigos na BVS, 228 na base PubMed e 100 no Google Scholar. Não foram recuperados artigos na base Scielo. Antes do rastreamento dos artigos, 11 foram excluídos por serem duplicados. Após a leitura dos títulos e resumos dos 365 remanescentes, foram excluídos 251 artigos que não correspondiam à temática de interesse. Dos 114 artigos restantes, 23 foram excluídos por não apresentarem texto completo disponível eletronicamente. Finalmente, após a leitura dos textos de forma integral, 64 estudos foram excluídos por não representarem fielmente a proposta desta revisão. Assim, 27 artigos perfazem o total da presente revisão integrativa (Gráfico 1).

Gráfico 1 - Fluxograma do processo de busca dos artigos, 2021.

\section{Publicações identificadas na pesquisa em bases de dados}

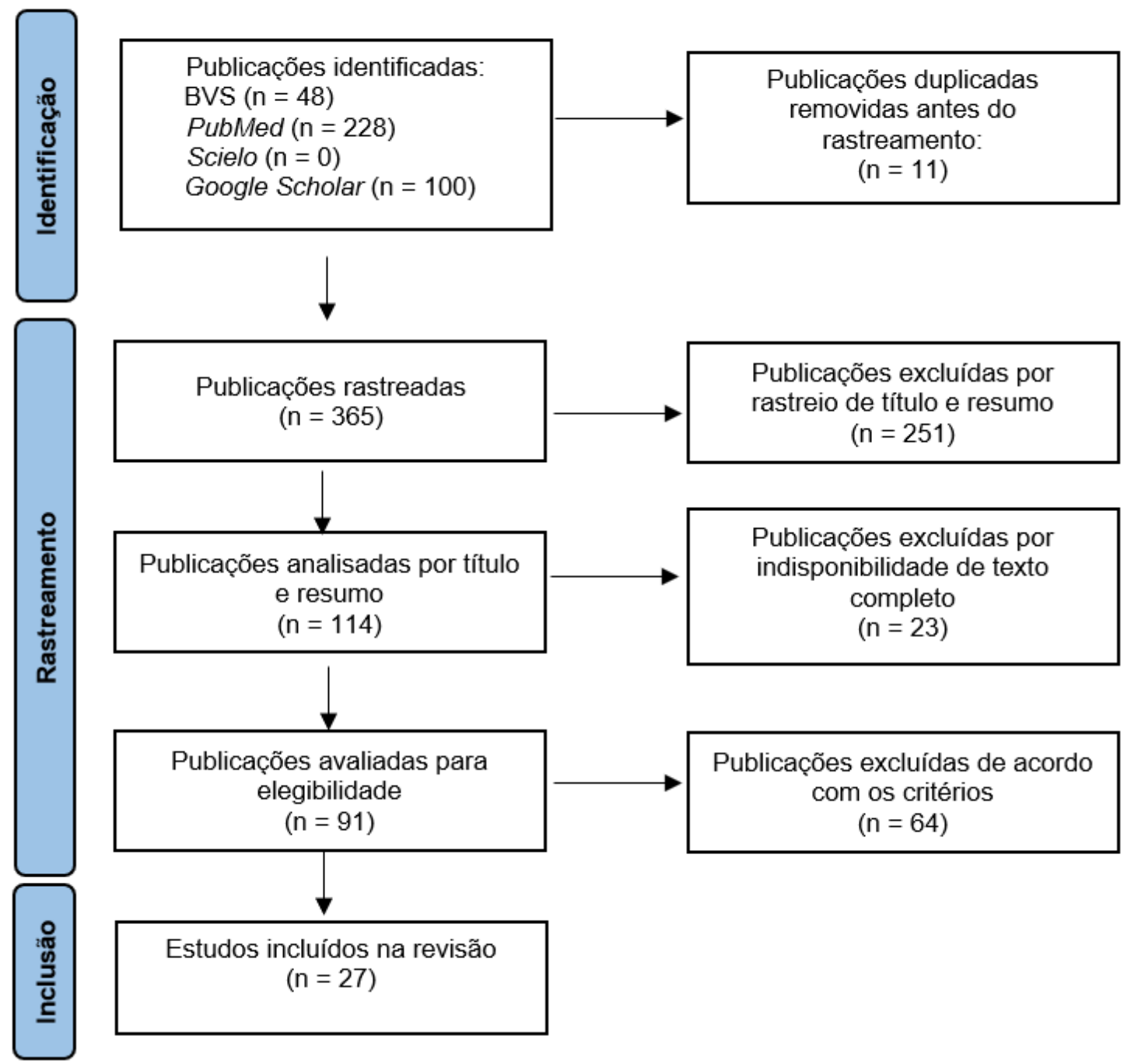

Fonte: PRISMA Flow Diagram.

Os estudos incluídos nesta revisão foram publicados no intervalo entre os anos de 2000 e 2020 . Com relação ao país de origem, verificou-se que a maior parte dos estudos foram do Canadá, com sete publicações (Archibald et al., 2018; Fogel, Khamisa, Afkham, Liddy, \& Keely, 2016; Johnston et al., 2017; Kohlert et al., 2018; Liddy et al., 2019b; Liddy, Moroz, Mihan, \& Keely, 2019c; Liddy et al., 2016b); seguidos pela Holanda (van den Akker et al., 2001; van der Heijden, de Keizer, Bos, Spuls, \& Witkamp, 2011; van der Velden et al., 2019; van Gelder et al., 2017), e EUA (Chittle et al., 2015; Gilani, Bommakanti, 
\& Friedman, 2020; Schettini et al., 2019), com quatro e três publicações respectivamente; Reino Unido (Mehrtens, Shall, \& Halpern, 2019; Stoves et al., 2010), Finlândia (Harno, Paavola, Carlson, \& Viikinkoski, 2000; Jaatinen, Aarnio, Remes, Hannukainen, \& Köymäri-Seilonen, 2002), Itália (Scalvini, Rivadossi, Comini, Muiesan, \& Glisenti, 2011; Scalvini et al., 2009), Brasil (Carrard et al., 2018; Katz et al., 2020) e Espanha (Lacasta Tintorer et al., 2020; Vidal-Alaball et al., 2018) sediando duas publicações; e finalmente, Portugal (Paiva et al., 2001), França (Piette, Nougairède, Vuong, Crickx, \& Tran, 2017) e Nova Zelândia (Mann \& van de Weijer, 2018) foram os países de origem de uma publicação cada um.

Quanto às áreas da saúde nas quais os sistemas eletrônicos de comunicação foram utilizados, cinco estudos se concentraram na especialidade de Dermatologia (Mehrtens et al., 2019; Piette et al., 2017; van den Akker et al., 2001; van der Heijden et al., 2011; Vidal-Alaball et al., 2018), três na área de Nefrologia (van Gelder et al., 2017; Schettini et al., 2019; Stoves et al., 2010), e dois nas áreas de Hematologia (Fogel et al., 2016; Johnston et al., 2017) e Otorrinolaringologia (Gilani et al., 2020; Kohlert et al., 2018); um estudo nas áreas de Neurologia (Paiva et al., 2001), Dor Crônica (Liddy et al., 2016), Estomatologia (Carrard et al., 2018), Psiquiatria (Archibald et al., 2018), Cardiologia (Scalvini et al., 2011), Angiologia (Chittle et al., 2015) e Ginecologia (Mann et al., 2018). Estudos reportando sistemas para múltiplas especialidades foram em número de oito (Harno et al., 2000; Jaatinen et al., 2002; Katz et al., 2020; Lacasta Tintorer et al., 2020; Liddy et al., 2019b; Liddy et al., 2019c; Scalvini et al., 2009; van der Velden et al., 2019).

Vinte e seis dos 27 estudos selecionados trataram de sistemas clássicos de consulta eletrônica, e um estudo (Katz et al., 2020) trouxe um relato de experiência de projeto vinculado ao Programa Nacional de Telessaúde.

O Quadro 1 sumariza os estudos selecionados nesta revisão.

Quadro 1 - Sumário das principais características dos artigos elencados na presente revisão integrativa, 2021.

\begin{tabular}{|c|c|c|c|}
\hline $\begin{array}{c}\text { Autor Principal/Ano } \\
\text { País } \\
\text { Sistema de Comunicação }\end{array}$ & $\begin{array}{l}\text { Consultas Eletrônicas } \\
\text { (n) } \\
\text { Desenho do Estudo }\end{array}$ & Objetivos & Conclusões \\
\hline $\begin{array}{c}\text { Harno et al./2000 } \\
\text { Finlândia } \\
\text { Plataforma web-based }\end{array}$ & Prospectivo comparativo & $\begin{array}{l}\text { Avaliar eficácia e custos } \\
\text { da telemedicina no } \\
\text { processo de referência } \\
\text { entre APS e AE, em dois } \\
\text { hospitais. }\end{array}$ & $\begin{array}{l}\text { Melhoria da eficácia e } \\
\text { produtividade na APS, com } \\
\text { redução de custos diretos no } \\
\text { processo de referência } \\
\text { tradicional. }\end{array}$ \\
\hline $\begin{array}{c}\text { Paiva } \text { et al./2001 } \\
\text { Portugal } \\
\text { Videoconferência médica }\end{array}$ & Transversal & $\begin{array}{l}\text { Implementar consultas } \\
\text { eletrônicas em } \\
\text { neurologia e avaliar os } \\
\text { resultados. }\end{array}$ & $\begin{array}{l}\text { Sucesso na implementação, com } \\
\text { apoio na gestão terapêutica e } \\
\text { diagnóstica de casos } \\
\text { neurológicos. }\end{array}$ \\
\hline $\begin{array}{c}\text { van den Akker } \text { et al./2001 } \\
\text { Holanda } \\
\text { Sistema de email }\end{array}$ & $\begin{array}{c}89 \\
\text { Transversal }\end{array}$ & $\begin{array}{l}\text { Avaliar viabilidade da } \\
\text { teledermatologia através } \\
\text { de um sistema de } e \text {-mail. }\end{array}$ & $\begin{array}{l}\text { Viabilidade no apoio ao } \\
\text { diagnóstico e planejamento } \\
\text { terapêutico. }\end{array}$ \\
\hline $\begin{array}{c}\text { Jaatinen } \text { et al./2002 } \\
\text { Finlândia } \\
\text { Plataforma web-based }\end{array}$ & Caso-controle & $\begin{array}{l}\text { Comparar um sistema } \\
\text { web-based de } \\
\text { encaminhamento da } \\
\text { APS para a AE, com o } \\
\text { sistema tradicional. }\end{array}$ & $\begin{array}{l}\text { Aumento da possibilidade da } \\
\text { APS responsabilizar-se pelo } \\
\text { tratamento, reduzindo a } \\
\text { necessidade de consultas } \\
\text { presenciais. }\end{array}$ \\
\hline $\begin{array}{c}\text { Scalvini et al./2009 } \\
\text { Itália } \\
\text { Telefonia móvel ou fixa + } \\
\text { plataforma web-based }\end{array}$ & Transversal & $\begin{array}{l}\text { Avaliar viabilidade de } \\
\text { um serviço de } \\
\text { telemedicina entre APS } \\
\text { e AE. }\end{array}$ & $\begin{array}{l}\text { Serviço viável. Redução de } 97 \% \\
\text { de encaminhamentos para a } \mathrm{AE} \\
\text { (área de cardiologia). }\end{array}$ \\
\hline $\begin{array}{l}\text { Stoves } \text { et al./2010 } \\
\text { Reino Unido } \\
\text { Plataforma web-based }+ \\
\text { prontuário eletrônico }\end{array}$ & $\begin{array}{l}\quad 68 \\
\text { Longitudinal } \\
\text { prospectivo }\end{array}$ & $\begin{array}{l}\text { Avaliar impactos de } \\
\text { consultas eletrônicas no } \\
\text { manejo de pacientes } \\
\text { renais crônicos. }\end{array}$ & $\begin{array}{l}\text { Gerenciamento eficaz da doença } \\
\text { renal crônica na APS (evitou } \\
\text { encaminhamento em } 84 \% \text { dos } \\
\text { casos discutidos). }\end{array}$ \\
\hline $\begin{array}{c}\text { van der Heijden et al./2011 } \\
\text { Holanda } \\
\text { Plataforma web-based }\end{array}$ & $\begin{array}{l}37.207 \\
\text { Coorte }\end{array}$ & $\begin{array}{l}\text { Investigar efeito da } \\
\text { teledermatologia, quanto }\end{array}$ & $\begin{array}{l}\text { Atendimento eficiente, com taxa } \\
\text { de encaminhamento evitado de }\end{array}$ \\
\hline
\end{tabular}




\begin{tabular}{|c|c|c|c|}
\hline & & $\begin{array}{l}\text { à eficiência, qualidade e } \\
\text { custos. }\end{array}$ & $\begin{array}{l}68 \% \text {, e redução de custos } \\
\text { estimada em } 18 \% \text {. }\end{array}$ \\
\hline $\begin{array}{l}\text { Scalvini } \text { et al./2011 } \\
\text { Itália } \\
\text { Telefonia móvel ou fixa }+ \\
\text { plataforma web-based }\end{array}$ & $\begin{array}{c}1.719 \\
\text { Observacional } \\
\text { prospectivo }\end{array}$ & $\begin{array}{l}\text { Avaliar utilidade da } \\
\text { teleconsulta no apoio ao } \\
\text { diagnóstico e manejo de } \\
\text { hipertensos. }\end{array}$ & $\begin{array}{l}\text { Melhoria da avaliação e manejo } \\
\text { de hipertensos em tempo real, } \\
\text { permitindo o acompanhamento } \\
\text { via teleconsulta na APS. }\end{array}$ \\
\hline $\begin{array}{l}\text { Chittle } \text { et al./2015 } \\
\text { EUA } \\
\text { Plataforma web-based + } \\
\text { prontuário eletrônico }\end{array}$ & $\begin{array}{l}54 \\
\text { Prospectivo (piloto) }\end{array}$ & $\begin{array}{l}\text { Avaliar satisfação, e } \\
\text { resultados de consultas } \\
\text { eletrônicas no manejo de } \\
\text { doenças vasculares. }\end{array}$ & $\begin{array}{l}\text { Redução de } 80 \% \text { nos } \\
\text { encaminhamentos para AE, com } \\
\text { altas taxas de satisfação. }\end{array}$ \\
\hline $\begin{array}{c}\text { Fogel } \text { et al./2016 } \\
\text { Canadá } \\
\text { Plataforma web-based }\end{array}$ & Transversal & $\begin{array}{l}\text { Analisar uso, conteúdo e } \\
\text { valor percebido de } \\
\text { consultas eletrônicas em } \\
\text { hematologia. }\end{array}$ & $\begin{array}{l}\text { Aumento do acesso aos cuidados } \\
\text { de hematologia. Economia de } \\
\text { custos e redução de } 46 \% \text { no } \\
\text { encaminhamento para a AE. }\end{array}$ \\
\hline $\begin{array}{c}\text { Liddy et al./2016 } \\
\text { Canadá } \\
\text { Plataforma web-based }\end{array}$ & Transversal & $\begin{array}{l}\text { Descrever impacto de } \\
\text { consultas eletrônicas no } \\
\text { acesso à AE na área de } \\
\text { dor crônica. }\end{array}$ & $\begin{array}{l}\text { Mitigou efeitos negativos de } \\
\text { longo tempo de espera para } \\
\text { consulta na AE. Evitou consulta } \\
\text { presencial em } 36 \% \text { dos casos. }\end{array}$ \\
\hline $\begin{array}{l}\text { Piette } \text { et al./2017 } \\
\text { França } \\
\text { Sistema de email }\end{array}$ & $\begin{array}{c}53 \\
\text { Estudo clínico } \\
\text { controlado randomizado }\end{array}$ & $\begin{array}{l}\text { Avaliar impacto da } \\
\text { teledermatologia no } \\
\text { tempo de espera para } \\
\text { início de tratamento. }\end{array}$ & $\begin{array}{l}\text { Redução significativa no atraso } \\
\text { antes de iniciar o tratamento. } \\
\text { Evitou consulta presencial em } \\
45,3 \% \text { dos casos. }\end{array}$ \\
\hline $\begin{array}{l}\text { Johnston et al./2017 } \\
\text { Canadá } \\
\text { Plataforma web-based }\end{array}$ & Transversal & $\begin{array}{l}\text { Descrever uso de } \\
\text { consultas eletrônicas em } \\
\text { hematologia / oncologia } \\
\text { pediátrica. }\end{array}$ & $\begin{array}{l}\text { Implementação e uso bem- } \\
\text { sucedidos, resultando em evitar } \\
40 \% \text { de consultas presenciais. }\end{array}$ \\
\hline $\begin{array}{l}\text { van Gelder } \text { et al./2017 } \\
\text { Holanda } \\
\text { Plataforma web-based + } \\
\text { prontuário eletrônico }\end{array}$ & $\begin{array}{c}1.149 \\
\text { Estudo clínico } \\
\text { controlado randomizado }\end{array}$ & $\begin{array}{l}\text { Investigar redução de } \\
\text { encaminhamento para a } \\
\text { AE por um sistema de } \\
\text { telenefrologia. }\end{array}$ & $\begin{array}{l}\text { Os dados não permitiram } \\
\text { conclusões acerca dos efeitos do } \\
\text { sistema nas taxas de } \\
\text { encaminhamentos para a } \mathrm{AE} \text {. }\end{array}$ \\
\hline $\begin{array}{l}\text { Kohlert } \text { et al./2018 } \\
\text { Canadá } \\
\text { Plataforma web-based }\end{array}$ & $\begin{array}{l}109 \\
\text { Transversal }\end{array}$ & $\begin{array}{l}\text { Avaliar viabilidade e } \\
\text { eficácia de consultas } \\
\text { eletrônicas em } \\
\text { otorrinolaringologia- } \\
\text { cirurgia de cabeça e } \\
\text { pescoço. }\end{array}$ & $\begin{array}{l}\text { Evitou encaminhamento } \\
\text { desnecessário em } 48,7 \% \text { dos } \\
\text { casos discutidos. Enfatizou-se o } \\
\text { potencial para reduzir tempo de } \\
\text { espera por consulta presencial. }\end{array}$ \\
\hline $\begin{array}{l}\text { Archibald et al./2018 } \\
\text { Canadá } \\
\text { Plataforma web-based }\end{array}$ & $\begin{array}{c}169 \\
\text { Transversal }\end{array}$ & $\begin{array}{l}\text { Analisar a eficácia de } \\
\text { um serviço de consultas } \\
\text { eletrônicas na área de } \\
\text { psiquiatria. }\end{array}$ & $\begin{array}{l}\text { Melhoria do manejo de doenças } \\
\text { mentais na APS. Evitou 30,7\% } \\
\text { de encaminhamento } \\
\text { desnecessário. }\end{array}$ \\
\hline $\begin{array}{l}\text { Carrard } \text { et al./2018 } \\
\text { Brasil } \\
\text { Plataforma web-based }\end{array}$ & Transversal & $\begin{array}{l}\text { Resumir a experiência } \\
\text { do programa } \\
\text { EstomatoNet, voltado } \\
\text { para profissionais da } \\
\text { APS no sul do Brasil. }\end{array}$ & $\begin{array}{l}\text { Programa viável e com potencial } \\
\text { para melhorar a qualidade } \\
\text { assistencial da APS. Redução de } \\
40 \% \text { na intenção de } \\
\text { encaminhamento para AE. }\end{array}$ \\
\hline $\begin{array}{c}\text { Mann } \text { et al./2018 } \\
\text { Nova Zelândia } \\
\text { Plataforma web-based }\end{array}$ & Estudo retrospectivo & $\begin{array}{l}\text { Descrever } \\
\text { desenvolvimento, } \\
\text { eficácia e segurança de } \\
\text { consultas eletrônicas na } \\
\text { área de ginecologia. }\end{array}$ & $\begin{array}{l}\text { Eficaz na redução do número de } \\
\text { encaminhamentos para avaliação } \\
\text { presencial (conseguiu-se dar } \\
\text { suporte na APS em } 18,2 \% \text { dos } \\
\text { casos discutidos). }\end{array}$ \\
\hline $\begin{array}{c}\text { Vidal-Alaball } \text { et al./2018 } \\
\text { Espanha } \\
\text { Plataforma web-based + } \\
\text { prontuário eletrônico }\end{array}$ & $\begin{array}{l}5.606 \\
\text { Estudo retrospectivo } \\
\text { (análise do banco de } \\
\text { dados de um serviço) }\end{array}$ & $\begin{array}{l}\text { Avaliar impacto } \\
\text { econômico dos serviços } \\
\text { de telemedicina na } \\
\text { região central da } \\
\text { Catalunha. }\end{array}$ & $\begin{array}{l}\text { A utilização de um serviço de } \\
\text { consultas eletrônicas em } \\
\text { dermatologia, em vez de } \\
\text { consultas presenciais, pôde } \\
\text { poupar } € 51.164 \text { por ano. }\end{array}$ \\
\hline $\begin{array}{l}\text { Schettini } \text { et al./2019 } \\
\text { EUA } \\
\text { Plataforma web-based }+ \\
\text { prontuário eletrônico }\end{array}$ & Coorte retrospectiva & $\begin{array}{l}\text { Caracterizar demanda } \\
\text { por consultas eletrônicas } \\
\text { em nefrologia e } \\
\text { benefícios ao processo } \\
\text { referencial. }\end{array}$ & $\begin{array}{l}\text { Evitaram-se encaminhamentos } \\
\text { em } 67.5 \% \text { dos casos. Redução } \\
\text { dos tempos de espera e aumento } \\
\text { das taxas de conclusão do } \\
\text { processo de referência. }\end{array}$ \\
\hline
\end{tabular}




\begin{tabular}{|c|c|c|c|}
\hline $\begin{array}{l}\text { Liddy } \text { et } \text { al./2019b } \\
\text { Canadá } \\
\text { Plataforma web-based }\end{array}$ & $96-6.885^{*}$ & $\begin{array}{l}\text { Analisar processo de } \\
\text { implementação e } \\
\text { impactos de um serviço } \\
\text { de consultas eletrônicas } \\
\text { em quatro províncias } \\
\text { canadenses. }\end{array}$ & $\begin{array}{l}\text { Consistência sobre aspecto } \\
\text { educacional e impacto na decisão } \\
\text { de encaminhamento. Taxas de } \\
\text { encaminhamentos evitados } \\
\text { variaram entre } 24 \% \text { e } 53 \% \text { entre } \\
\text { as regiões estudadas. }\end{array}$ \\
\hline $\begin{array}{l}\text { Liddy } \text { et } \text { al./2019c } \\
\text { Canadá } \\
\text { Plataforma web-based }\end{array}$ & $\begin{array}{c}301 \\
\text { Longitudinal } \\
\text { prospectivo quali- } \\
\text { quantitativo }\end{array}$ & $\begin{array}{l}\text { Avaliar impactos de um } \\
\text { serviço de consultas } \\
\text { eletrônicas em uma } \\
\text { região de Ontário. }\end{array}$ & $\begin{array}{l}\text { Consulta presencial com o } \\
\text { especialista foi evitada em } 40 \% \\
\text { de todos os casos discutidos por } \\
\text { consulta eletrônica. }\end{array}$ \\
\hline $\begin{array}{c}\text { van der Velden } \text { et al./2019 } \\
\text { Holanda } \\
\text { Plataforma web-based }\end{array}$ & 112 & $\begin{array}{l}\text { Examinar viabilidade e } \\
\text { custos de um serviço de } \\
\text { consulta eletrônica } \\
\text { multiespecialidades. }\end{array}$ & $\begin{array}{l}\text { Serviço viável, com potencial de } \\
\text { reduzir quase metade de todos os } \\
\text { encaminhamentos para a } \mathrm{AE} \text {. } \\
\text { Economia de custos. }\end{array}$ \\
\hline $\begin{array}{l}\text { Mehrtens et al./2019 } \\
\text { Reino Unido } \\
\text { Plataforma web-based }\end{array}$ & $\begin{array}{l}40.201 \\
\text { Estudo retrospectivo } \\
\text { (análise do banco de } \\
\text { dados de um serviço) }\end{array}$ & $\begin{array}{l}\text { Avaliar um serviço de } \\
\text { teledermatologia, em } n^{\mathbf{o}} \\
\text { de consultas eletrônicas } \\
\text { realizadas, diagnósticos } \\
\text { e nível de satisfação. }\end{array}$ & $\begin{array}{l}\text { Serviço evitou } 16.282 \\
\text { encaminhamentos em } 14 \text { anos. } \\
\text { Diagnósticos principais foram } \\
\text { lesões dermatológicas. Bons } \\
\text { níveis de satisfação de usuários. }\end{array}$ \\
\hline $\begin{array}{c}\text { Gilani } \text { et al./2020 } \\
\text { EUA } \\
\text { Plataforma web-based }\end{array}$ & $\begin{array}{c}64 \\
\text { Transversal }\end{array}$ & $\begin{array}{l}\text { Investigar motivos de } \\
\text { consultas eletrônicas em } \\
\text { otorrinolaringologia e } \\
\text { eficácia do sistema. }\end{array}$ & $\begin{array}{l}\text { Uso principal no apoio ao manejo } \\
\text { de condições otológicas. Evitou- } \\
\text { se encaminhamento em } 82,8 \% \\
\text { dos casos discutidos. }\end{array}$ \\
\hline $\begin{array}{c}\text { Lacasta Tintorer et al./2020 } \\
\text { Espanha } \\
\text { Plataforma web-based }\end{array}$ & Estudo comparativo & $\begin{array}{l}\text { Analisar se uma } \\
\text { plataforma de } \\
\text { comunicação entre } \\
\text { médicos reduz } \\
\text { encaminhamentos. }\end{array}$ & $\begin{array}{l}\text { O uso de uma comunidade de } \\
\text { prática clínica por profissionais } \\
\text { da APS e especialistas ajudou a } \\
\text { reduzir o número de } \\
\text { encaminhamentos para a AE. }\end{array}$ \\
\hline $\begin{array}{c}\text { Katz et al./2020 } \\
\text { Brasil } \\
\text { Plataforma web-based }\end{array}$ & $\begin{array}{l}\text { Estudo longitudinal } \\
\text { prospectivo }\end{array}$ & $\begin{array}{l}\text { Descrever os efeitos do } \\
\text { Projeto RegulaSUS na } \\
\text { APS e no acesso ao } \\
\text { cuidado especializado. }\end{array}$ & $\begin{array}{l}\text { Potencial na integração de } \\
\text { sistemas de saúde, impactando na } \\
\text { redução geral das filas de espera } \\
\text { da ordem de } 30 \% \text { para consultas } \\
\text { especializadas. }\end{array}$ \\
\hline
\end{tabular}

Legenda: *variação entre as quatro províncias estudadas (Alberta, Manitoba, Quebec, Newfoundland and Labrador) Fonte: Autores.

\section{Discussão}

De acordo com os artigos incluídos nesta revisão, sistemas eletrônicos de comunicação entre profissionais trouxeram importantes repercussões de cunho assistencial propriamente dito, mas também sob aspectos financeiros vinculados aos serviços de saúde. Na prática cotidiana de profissionais generalistas, as consultas eletrônicas obtiveram bons índices de satisfação tanto destes quanto dos pacientes envolvidos no processo, e foram capazes de aumentar a capacidade resolutiva da atenção primária, reduzindo encaminhamentos e tempo de espera para consultas na AE. A economia de custos experimentada com a estratégia, ao reduzir a necessidade de consultas presencias com especialistas nas mais diversas áreas da medicina, refletiu tanto sobre as fontes pagadoras dos serviços (poder público, seguradoras de saúde, etc), quanto sobre os gastos com deslocamentos pelos pacientes.

Ao longo dos últimos anos, serviços de consultas eletrônicas expandiram-se internacionalmente de forma significativa, mostrando-se eficazes em garantir acesso mais rápido ao aconselhamento de especialistas, propiciando impactos positivos sobre a qualidade da assistência em saúde prestada pela APS (Liddy, Moroz, Mihan, Nawar, \& Keely, 2019d; Joschko et al., 2018). As iniciativas diferem em questões estruturais e quanto aos estágios de implementação, com alguns serviços plenamente estabelecidos e outros em fase piloto (Joschko et al., 2018). Por tratar-se de uma inovação na prestação de cuidados de saúde, a disseminação generalizada deste tipo de serviço está na dependência da demonstração abrangente de seu valor, cujos impactos positivos devem ser óbvios e a qualidade da assistência aceitável para toda a população (Tuot et al., 2018). 
A redução no fluxo de encaminhamentos para a AE mediada pela estratégia de consulta eletrônica foi evidenciada em todos os 21 estudos desta revisão, com oscilações percentuais relacionadas a variáveis como tipo de especialidade incluída na abordagem, duração de tempo de análise do estudo e familiaridade prévia dos profissionais da APS com este tipo de estratégia, fatores que podem, decisivamente, influenciar na decisão de adoção e uso desses sistemas. No cenário dos estudos, os profissionais generalistas utilizaram-se da consulta eletrônica de forma voluntária, logo, o entendimento e a percepção de valor deste tipo de serviço sobre as práticas cotidianas, no sentido de ampliação de seu escopo assistencial, são fatores com potencial de estimular este uso. Outro ponto discutido como influenciador na decisão dos profissionais na utilização da estratégia, foi a percepção de que este tipo de serviço é eficaz para reduzir custos, ao permitir um diagnóstico correto, mais rápido, com redução do esforço e do custo financeiro envolvidos tanto para os médicos quanto para as instituições de saúde (Lacasta Tintorer et al., 2020).

No Brasil, em que pese o subfinanciamento crônico do sistema público de saúde, a capilaridade e capacidade já instalada do Programa Nacional de Telessaúde, concebido para ampliar a integração dos serviços assistenciais e melhorar a resolutividade da atenção primária, são elementos importantes no auxílio à regulação de encaminhamentos e gestão dos fluxos de pacientes entre os níveis que compõem estes serviços (Ministério da Saúde, n.d.). A teleconsultoria, atividade principal ofertada pelo Programa, traz consigo todo um potencial de aprimoramento das atividades desenvolvidas na atenção básica, ainda que não funcione nos moldes tradicionais de uma consulta eletrônica, uma vez que se destina a promover uma comunicação entre profissionais atuantes no ambiente da APS (sejam eles médicos, enfermeiros, agentes comunitários de saúde ou gestores) e uma equipe de profissionais especializada em atenção primária.

Reduzir o distanciamento e aproximar os especialistas e profissionais da APS são tidas como ações relevantes para a construção de uma atenção integral em saúde, e vêm sendo discutidas nos últimos anos com vistas a criar uma cultura de colaboração entre a APS e a AE (Almeida et al., 2013). O papel da APS como coordenadora do cuidado pode ser aprimorado mediante a integração de ações de telessaúde e regulação de pedidos de encaminhamento para a AE, conforme evidências do estudo brasileiro conduzido por Katz et al (2020), onde demonstrou-se uma otimização do trabalho na atenção primária e ao mesmo tempo, uma racionalização da demanda para consultas com especialistas.

Pela natureza de um serviço de cuidados primários exigir o manejo de condições clínicas diversas e em diferentes faixas etárias, é natural inferir que o profissional ali atuante necessite de um suporte especializado na condução clínica de determinados casos. Neste sentido, a troca de informações entre generalistas e especialistas mediada por um sistema de consulta eletrônica atuando como um suporte à tomada de decisão, aprimora a qualidade da assistência prestada pelos generalistas, e aumenta a eficiência e resolutividade deste primeiro nível de atenção à saúde. O valor educacional dos serviços de consulta eletrônica e os altos níveis de satisfação geral dos profissionais envolvidos com este tipo de estratégia de interação entre pares, foram parâmetros que emergiram a partir desta revisão, sendo também reconhecidos em análises conduzidas acerca de características de sistemas de consulta eletrônica europeus e norte-americanos (Tuot et al., 2018; Joschko et al., 2018).

$\mathrm{O}$ aspecto de redução de custos com estratégias de consulta eletrônica foi reconhecido em diversos trabalhos desta revisão (Archibald et al., 2018; Carrard et al., 2018; Fogel et al., 2016; Jaatinen et al., 2002; Kohlert et al., 2018; Lacasta Tintorer et al., 2020; Paiva et al., 2001; Schettini et al., 2019; Stoves et al., 2010; van den Akker et al., 2001), muito embora apenas seis dos 27 estudos elencados no total (Harno et al., 2000; Scalvini et al., 2009; van der Heijden et al., 2011; van der Velden et al., 2019; van Gelder et al., 2017; Vidal-Alaball et al., 2018) tenham realizado avaliações específicas acerca dos impactos econômicos da estratégia. Apesar de ainda escassos na literatura, estudos desta natureza, em geral, produzem resultados promissores em termos de redução de custos para os sistemas de saúde, retorno do investimento e otimização de recursos para a sociedade como um todo (Liddy et al., 2019d). 
As potencialidades de integração de sistemas de saúde geradas pelas novas tecnologias, a exemplo das plataformas para consultas eletrônicas, são inequívocas e trazem consigo perspectivas de melhoria dos desfechos na transição de cuidados da APS para a AE. Modelos inovadores para favorecer o processo de comunicação nos tempos atuais, os sistemas de consultas eletrônicas constituem uma abordagem bastante eficaz na regulação de encaminhamentos e no aprimoramento do acesso a consultas especializadas, reconhecidamente grandes desafios dos sistemas de saúde contemporâneos. Para além das vantagens citadas, destaca-se a necessidade de execução de estudos no âmbito econômico, com análise de custos vinculada às consultas eletrônicas, aspecto mencionado como relevante em diversos artigos desta revisão, mas pouco explorado na literatura.

Ressaltam-se como limitações deste estudo a busca sem recuperação de artigos na base Scielo e a ausência de avaliação da qualidade metodológica dos estudos selecionados.

\section{Conclusão}

Com diferentes objetivos e por variadas abordagens, todos os estudos integrantes desta revisão reportaram que a utilização de sistemas eletrônicos de comunicação entre especialistas e profissionais da atenção primária proporcionou uma redução nas taxas de encaminhamento para os serviços de atenção especializada, variando percentualmente de acordo com a área da medicina estudada. Consequentemente, filas de espera para consultas com especialistas são também reduzidas, bem como, avaliações presenciais são oportunizadas para os casos que efetivamente as necessitam. Ademais, um menor tempo de espera para obtenção do parecer clínico de um especialista é capaz de impactar positivamente na qualidade da assistência prestada no serviço de atenção primária, repercutindo na eficiência do tratamento e na qualidade de vida do paciente.

Abrem-se perspectivas futuras de estudos comparativos em relação à eficácia das diferentes abordagens ou mesmo do uso de sistemas eletrônicos de comunicação de forma complementar e concomitante à abordagem presencial. Além disso, a incorporação de outras tecnologias que modifiquem a relação entre os pacientes na atenção primária e os provedores da atenção especializada, e como os diversos atores percebem a inserção das tecnologias, podem variar na dependência do perfil de cada comunidade, motivando estudos específicos.

\section{Referências}

Almeida, P. F., Gérvas, J., Freire, J. M. \& Giovanella, L. (2013). Estratégias de integração entre atenção primária à saúde e atenção especializada: paralelos entre Brasil e Espanha. Saúde debate, 37(98), 400-415.

Archibald, D., Stratton, J., Liddy, C., Grant, R. E., Green, D. \& Keely, E. J. (2018). Evaluation of an electronic consultation service in psychiatry for primary care providers. BMC Psychiatry, 18, 119.

Belber, G. S., Passos, V. C. S., Borysow, I. C. \& Maeyama, M. A. (2021). Contribuições do Programa Nacional Telessaúde Brasil Redes na formação de recursos humanos na atenção básica. Braz J of Develop, 7(1), 1198-1219.

Bernardino Junior, S. V., Medeiros, C. R. G., Souza, C. F., Kich, J., Alves, A. M. \& Castro, L. C. (2020). Processos de encaminhamento a serviços especializados em cardiologia e endocrinologia pela Atenção Primária à Saúde. Saúde debate, 44(126), 694-707.

Botelho, L. L. R. B., Cunha, C. C. A. C. \& Macedo, M. (2011). O método da revisão integrativa nos estudos organizacionais. Gestão e Sociedade, 5(11), 121136.

Carrard, V. C., Roxo Gonçalves, M., Rodriguez Strey, J., Pilz, C., Martins, M., Martins, M. D., Schmitz, C. A., Dal Moro, R. G., D’Ávila, O. P., Drv Rados, Harzheim, E. \& Gonçalves, M. R. (2018). Telediagnosis of oral lesions in primary care: The EstomatoNet Program. Oral Dis, $24(6)$, $1012-1019$.

Chittle, M. D., Rao, S. K., Jaff, M. R., Patel, V. I., Gallen, K. M., Avadhani, R., Ferris, T. G. \& Wasfy, J. H. (2015). Asynchronous vascular consultation via electronic methods: A feasibility pilot. Vasc Med, 20(6), 551-556.

Farias, C. M. L., Giovanella, L., Oliveira, A. E. \& Santos Neto, E. T. (2019). Tempo de espera e absenteísmo na atenção especializada: um desafio para os sistemas universais de saúde. Saúde debate, 43(spe5), 190-204.

Fogel, A., Khamisa, K., Afkham, A., Liddy, C. \& Keely, E. (2016). Ask the eConsultant: Improving access to haematology expertise using an asynchronous eConsult system. J Telemed Telecare, 23(3), 421-427.

Gilani, S., Bommakanti, K. \& Friedman, L. (2020). Electronic Consults in Otolaryngology: A Pilot Study to Evaluate the Use, Content, and Outcomes in an Academic Health System. Ann Otol Rhinol Laryngol, 129 (2), 170-174. 
Harno, K., Paavola, T., Carlson, C. \& Viikinkoski, P. (2000). Patient referral by telemedicine: effectiveness and cost analysis of an intranet system. J Telemed Telecare, 6(6), 320-329.

Jaatinen, P. T., Aarnio, P., Remes, J., Hannukainen, J. \& Köymäri-Seilonen, T. (2002). Teleconsultation as a replacement for referral to an outpatient clinic. $J$ Telemed Telecare, 8(2), 102-106.

Johnston, D. L., Murto, K., Kurzawa, J., Liddy, C., Keely, E. \& Lai, L. (2017). Use of Electronic Consultation System to Improve Access to Care in Pediatric Hematology/Oncology. J Pediatr Hematol Oncol, 39(7), e367-e369.

Joschko, J., Keely, E., Grant, R., Moroz, I., Graveline, M., Drimer, N. \& Liddy, C. (2018). Electronic Consultation Services Worldwide: Environmental Scan. J Med Internet Res, 20(12), e11112.

Katz, N., Roman, R., Rados, D. V., Oliveira, E. B., Schmitz, C. A. A., Gonçalves, M. R., Mengue, S. S. \& Umpierre, R. N. (2020). Acesso e regulação ao cuidado especializado no Rio Grande do Sul: a estratégia RegulaSUS do TelessaúdeRS-UFRGS. Ciênc. saúde coletiva, 25(4), 1389-1400.

Knox, M., Murphy, E. J., Leslie, T., Wick, R. \& Tuot, D. S. (2020). e-Consult implementation success: lessons from 5 county-based delivery systems. Am J Manag Care, 26(1), e21-e27.

Kohlert, S., Murphy, P., Tse, D., Liddy, C., Afkham, A. \& Keely, E. (2018). Improving access to otolaryngology-head and neck surgery expert advice through eConsultations. Laryngoscope, 128(2), 350-355.

Lacasta Tintorer, D., Manresa Domínguez, J. M., Jiménez-Zarco, A., Rodríguez-Blanco, T., Flayeh Beneyto, S., Torán-Monserrat, P., Tuduri, X. M. \& SaigíRubió, F. (2020). Efficiency as a determinant of loyalty among users of a Community of Clinical Practice: a comparative study between the implementation and consolidation phases. BMC Fam Pract, 21(1), 15.

Liddy, C., Bello, A., Cook, J., Drimer, N., Pilon, M. D., Farrell, G., Glassford, J., Ireland, L., McDonald, R., Nabelsi, V., Oppenheimer, L., Singer, A. \& Keely, E. (2019b). Supporting the spread and scale-up of electronic consultation across Canada: cross-sectional analysis. BMJ Open, 9(5).

Liddy, C., Drosinis, P., Deri Armstrong, C., McKellips, F., Afkham, A. \& Keely, E. (2016a). What are the cost savings associated with providing access to specialist care through the Champlain BASE eConsult service? A costing evaluation. BMJ open, 6(6), e010920.

Liddy, C., Moroz, I., Keely, E., Taljaard, M., Deri Armstrong, C., Afkham, A. \& Kendall, C. E. (2019a). Understanding the impact of a multispecialty electronic consultation service on family physician referral rates to specialists: a randomized controlled trial using health administrative data. Trials, 20(1), 348.

Liddy, C., Moroz, I., Mihan, A. \& Keely, E. (2019c). Assessment of the Generalizability of an eConsult Service through Implementation in a New Health Region. J Am Board Fam Med, 32(2), 146-157.

Liddy, C., Moroz, I., Mihan, A., Nawar, N. \& Keely, E. (2019d). A Systematic Review of Asynchronous, Provider-to-Provider, Electronic Consultation Services to Improve Access to Specialty Care Available Worldwide. Telemed J E Health, 25(3), 184-198.

Liddy, C., Smyth, C., Poulin, P. A., Joschko, J., Rebelo, M. \& Keely, E. (2016b). Improving Access to Chronic Pain Services Through eConsultation: A CrossSectional Study of the Champlain BASE eConsult Service. Pain Med, 17(6), 1049-1057.

Mann, R. \& van de Weijer, P. H. M. (2018). Adopting innovation in gynaecology: The introduction of e-consult. Aust N Z J Obstet Gynaecol, 58(4), 449-453.

Mehrtens, S. H., Shall, L. \& Halpern, S. M. (2019). A 14-year review of a UK teledermatology service: experience of over 40000 teleconsultations. Clin Exp Dermatol, 44(8), 874-881.

Ministério da Saúde. (n.d.). Saúde Digital e Telessaúde. Página inicial. https://saudedigital.saude.gov.br/telessaude/

Olayiwola, J. N., Potapov, A., Gordon, A., Jurado, J., Magana, C., Knox, M. \& Tuot, D. (2019). Electronic consultation impact from the primary care clinician perspective: Outcomes from a national sample. J Telemed Telecare, 25(8), 493-498.

Organização Pan-Americana da Saúde. (2011). A atenção à saúde coordenada pela APS: construindo as redes de atenção no SUS: contribuições para o debate. Brasília: Organização Pan-Americana da Saúde. https://iris.paho.org/handle/10665.2/18457

Paiva, T., Coelho, H., Araújo, M. T., Rodrigues, R., Almeida, A., Navarro, T., Cruz, M., Carneiro, G. \& Belo, C. (2001). Neurological teleconsultation for general practitioners. J Telemed Telecare, 7(3), 149-54.

Piette, E., Nougairède, M., Vuong, V., Crickx, B. \& Tran, V-T. (2017). Impact of a store-and-forward teledermatology intervention versus usual care on delay before beginning treatment: A pragmatic cluster-randomized trial in ambulatory care. J Telemed Telecare, 23(8), 725-732.

Ray, K. N. \& Kahn, J. M. (2020). Connected Subspecialty Care: Applying Telehealth Strategies to Specific Referral Barriers. Acad Pediatr, 20(1), 16-22.

Scalvini, S., Rivadossi, F., Comini, L., Muiesan, M. L. \& Glisenti, F. (2011). Telemedicine: The role of specialist second opinion for GPs in the care of hypertensive patients. Blood Press, 20(3), 158-165.

Scalvini, S., Tridico, C., Glisenti, F., Giordano, A., Pirini, S., Peduzzi, P. \& Auxilia, F. (2009). The SUMMA Project: A Feasibility Study on Telemedicine in Selected Italian Areas. Telemed $j$ e. health, 15(3), 261-269.

Schettini, P., Shah, K. P., O’Leary, C. P., Patel, M. P., Anderson, J. B., Cho, A. H., Long, A. L., Bosworth, H. B. \& Blake Cameron, C. (2019). Keeping care connected: e-Consultation program improves access to nephrology care. J Telemed Telecare, 25(3), 142-150.

Stoves, J., Connolly, J., Cheung, C. K., Grange, A., Rhodes, P., O’Donoghue, D. \& Wright, J. (2010). Electronic consultation as an alternative to hospital referral for patients with chronic kidney disease: a novel application for networked electronic health records to improve the accessibility and efficiency of healthcare. Qual Saf Health Care, 19(5), e54. 
Tuot, D. S., Liddy, C., Vimalananda, V. G., Pecina, J., Murphy, E. J., Keely, E., Simon, S. R., North, F., Orlander, J. D. \& Chen, A. H. (2018). Evaluating diverse electronic consultation programs with a common framework. BMC health services research, 18(1), 814.

van den Akker, T. W., Reker, C. H., Knol, A., Post, J., Wilbrink, J. \& van der Veen, J. P. (2001). Teledermatology as a tool for communication between general practitioners and dermatologists. J Telemed Telecare, 7(4), 193-8.

van der Heijden, J. P., de Keizer, N. F., Bos, J. D., Spuls, P. I. \& Witkamp, L. (2011). Teledermatology applied following patient selection by general practitioners in daily practice improves efficiency and quality of care at lower cost. Br J Dermatol, 165(5), 1058-65.

van der Velden, T., Schalk, B. W. M., Harmsen, M., Adriaansens, G., Schermer, T. R. \& Ten Dam, M. A. (2019). Implementation of web-based hospital specialist consultations to improve quality and expediency of general practitioners' care: a feasibility study. BMC Fam Pract, $20(1), 73$.

van Gelder, V. A., Scherpbier-de Haan, N. D., van Berkel, S., Akkermans, R. P., de Grauw, I. S., Adang, E. M., Assendelft, P. J., de Grauw. W. J. C., Biermans, M. C. J. \& Wetzels, J. F. M. (2017). Web-based consultation between general practitioners and nephrologists: a cluster randomized controlled trial. Fam Pract, $34(4), 430-436$

Vidal-Alaball, J., Garcia Domingo, J. L., Garcia Cuyàs, F., Mendioroz Peña, J., Flores Mateo, G., Deniel Rosanas, J. \& Valmaña, G. S. (2018). A cost savings analysis of asynchronous teledermatology compared to face-to-face dermatology in Catalonia. BMC Health Serv Res, 18(1), 650. 